\title{
Anorectal malformation as a cause of recurring perineal abscesses - value of anorectal endosonography and magnetic resonance imaging: a case report
}

\author{
Małgorzata Kołodziejczak ${ }^{1}$, Giulio A Santoro ${ }^{2}$, Jacek Sobocki ${ }^{3}$, Tomasz Szopiński ${ }^{4}$, \\ Anna Wiączek ${ }^{1,5}$, Iwona Sudoł-Szopińska ${ }^{6,7}$
}

${ }^{1}$ Warsaw Proctology Centre, Saint Elisabeth Hospital, Warsaw, Poland, ${ }^{2}$ Department of Colorectal Surgery, Digestive Disease Institute, Cleveland Clinic Abu Dhabi, Abu Dhabi, UAE, ${ }^{3}$ Clinic of Nutrition and Surgery, Orłowski Hospital in Warsaw, Poland, ${ }^{4}$ Department of Urology of the Jagiellonian University, Collegium Medicum, Krakow, Poland, ${ }^{5}$ General Surgical Unit, Solec Hospital in Warsaw, Poland, ${ }^{6}$ Radiology Department, National Institute of Geriatrics, Rheumatology and Rehabilitation, Warsaw, Poland, ${ }^{7}$ Imaging Department of the Second Faculty of Medicine, Warsaw Medical University, Warsaw, Poland

\begin{abstract}
Malformations of the rectum and urinary tract frequently coexist, and the prevalence of urogenital defects in patients with a rectal defect ranges from 20 to $54 \%$. In most patients, anorectal malformations are diagnosed and treated surgically in early childhood. In this report, we present a case of a 52-year-old male with a history of urological operations in infancy due to a congenital urethral malformation and multiple recurrent episodes of perineal abscesses with urinary retention. Anorectal endosonography and magnetic resonance imaging revealed the presence of a large cystic lesion adjacent to the rectal wall which became smaller at the level of the puborectalis. The walls of the lesion had a layered structure identical to the wall of the rectum. In addition, the examinations showed hypotrophy of the left lobe of the prostate and lack of left semnal vesicles. Thus, the lesion was diagnosed as duplication of the rectum or rectal diverticulum. Surgery was performed by an interdisciplinary team of colorectal surgeons and urologist. The lesion was removed laparoscopically. The pathological report revealed that the cystic wall was typical of the intestinal wall, consistent with a diagnosis of rectal duplication.
\end{abstract}

Keywords: anorectal endosonography, transrectal sonography, rectal duplication, rectal malformation

\section{Introduction}

Anorectal malformations can be complex issues. They may occur during the fifth to seventh weeks of fetal development in approximately 1:2500 births. With these defects, the anus (opening at the end of the large intestine through which stool passes) and the rectum (area of the large intestine just above the anus) do not develop

Received 25.07.2016 Accepted 24.08.2016

Med Ultrason

2017, Vol. 19, No 2, 228-231

Corresponding author: Iwona Sudoł-Szopińska

Radiology Department, Institute of

Rheumatology

Imaging Department of the Second Faculty of

Medicine, Warsaw Medical University,

Warsaw, Poland

E-mail: sudolszopinska@gmail.com properly. Abnormalities of the rectum and urinary tract coexist in $20-54 \%$ of cases [1,2] caused by embryonic aberration of the uro-rectal septum. In most patients, anorectal defects are treated surgically in early childhood. We present a case of an adult patient with recurring anal abscesses due to a complex rectal malformation.

\section{Case report}

A 52-year-old patient presented to the Emergency Department complaining of anal pain for recurrent perineal abscess and urinary retention. At history, he had had multiple urological operations in infancy to treat a congenital urethral defect and had cystostomy performed several times because of urethral stricture. He also had undergone 12 incision and drainage of perineal abscesses. At physical examination, a left side perianal abscess was found. 


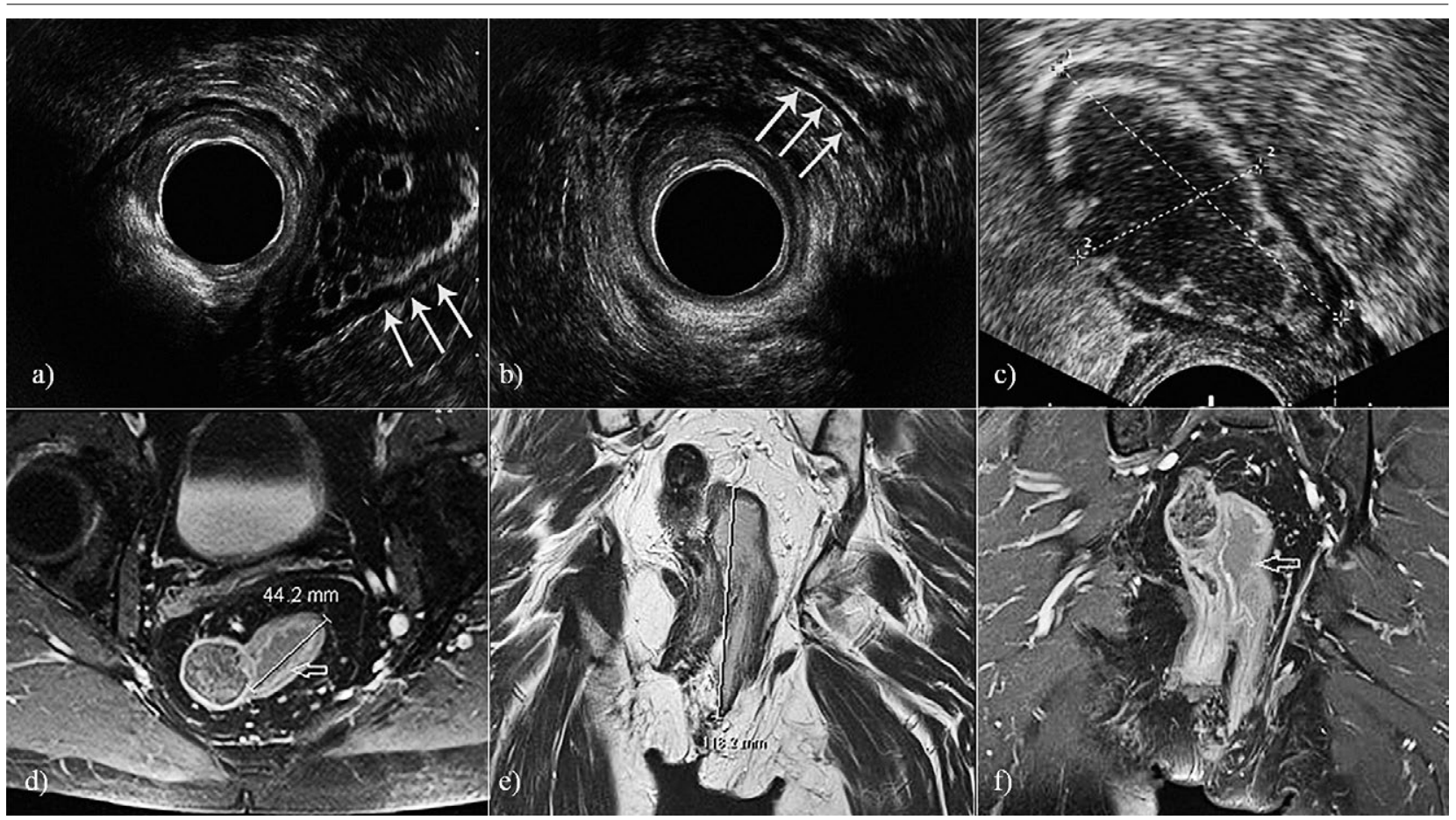

Fig 1. Endosonography of the anal canal and rectum and pelvic MRI: a) a cystic lesion, with diameters 47 x 25 (transverse plane of imaging), was identified at 10-12 cm from the anal margin, in direct continuity with the left rectal wall, and b) became smaller at the PR level. The cystic wall had the same five layers of the rectal wall, and was diagnosed as rectal diverticulum or duplication. There were no signs of communication with the rectal lumen; the lesion was also well seen in ERUS examination (c); T1FSCE images in the transverse plane (d), T2 (e) and TIRM images (f) in the coronal plane. A lesion with fluid content was visible adjacent to the left rectal wall. The lesion adheres to the left rectal wall. The wall structure of the lesion is identical to the rectum. Arrows- mucosa folds in the lesion.

Scars from previous incisions were present in the soft tissues of the left buttock. He was admitted to the Colorectal Department. Cystostomy was positioned for the urinary retention and the anal suppurative collection was drained. Patient's condition after the procedure immediately improved and a diagnostic work-up with endorectal ultrasonography (ERUS), magnetic resonance imaging (MRI) of the pelvis and colonoscopy was performed.

ERUS was performed with a BK Medical Profocus and a 360 rotating transducer with 3D automatic acquisition, at $16 \mathrm{MHz}$ frequency (type 2050, BK Medical Analogic) [3]. At ultrasound, on the left side of the rectum, a fluid collection, $60 \mathrm{~mm} \times 43 \mathrm{~mm}$ in size, was identified at $10-12 \mathrm{~cm}$ from the anal margin. It appeared like a cyst and the characteristic of its wall was identical to the five-layer structure of the rectum (fig 1a-c). There were no signs of peristalsis into the lesion and there was no evidence of communication with the rectum, but only continuity for several centimetres. From the US findings the differential diagnosis was between a rectal duplication and rectal diverticulum. On the left side of the anal canal, there was a subcutaneous fistula originating from the cystic lesion.
MRI was performed using a Siemens $1.5 \mathrm{~T}$ scanner in T2, T2 TIRM and T1FS sequences before and after administration of a contrast agent in coronal, transverse and sagittal planes. On the left side of the pelvis, a large fluid lesion (40x25 $\mathrm{mm}$ in the transverse plane; length $110 \mathrm{~mm}$ ) adjacent to the rectum was visualised. The structure of its wall was morphologically similar to the rectal wall (fig 1d-f). A diagnosis of a large diverticulum with possible neck localised within the left wall of the rectal ampulla was supposed, but not confirmed for the absence of a clear communication. There was also a left perianal fistula originating from the lesion. The left branch of the puborectalis muscle was deformed and the posterior part of this muscle was shifted laterally. Moreover, prostate asymmetry (smaller left lobe) and the absence of the left seminal vesicle were also detected. These findings suggested developmental abnormalities.

At colonoscopy the anterior and left lateral rectal wall appeared to be compressed at $7 \mathrm{~cm}$ from the anal margin, from an external mass. The mucosa was normal. No internal opening of diverticulum was identified, as well as no internal opening of the perianal fistula was found. 
After the diagnostic work-up, the patient was scheduled for laparoscopic surgery conducted by an interdisciplinary team including colorectal surgeon and urologist. A cystic lesion adjacent to the anterior rectal wall was identified. Blunt dissection could not be performed because of the absence of a plane between the two structures. Therefore, the lesion was incised and dense yellowish content, which was atypical for purulent fluid, was drained. The fistulous tract, running towards the buttock, was identified and excised. The patient was discharged home after 7 days in a good health condition. The histopathological report of the cystic lesion demonstrated the presence of a retained glandular structure, with chronic inflammation, hyperaemia and oedema of the mucosa, lymphatic nodules, and thickened muscle layer. The diagnosis of rectal duplication was confirmed.

\section{Discussions}

The incidence of anorectal defects is 1 per 5.000 births [4]. In most patients, anorectal and urogenital malformations are diagnosed and treated surgically in early childhood. Therefore, to diagnose and treat a congenital rectal defect in the adult, can be challenging. We presented a case of a 52-year old male suffering from recurrent perineal abscesses for many years. The history of a surgery to the urinary tract in early childhood, the concomitant urinary retention due to urethral stricture with the necessity to perform a cystostomy, and the absence of the left seminal vesicles, and the hypoplasia of the left lobe of the prostate oriented towards a complex rectal and urogenital malformation.

Defects of these two systems frequently coexist $[1,2]$. Anorectal malformations develop in the first trimester of pregnancy, usually between the $4^{\text {th }}$ and $6^{\text {th }}$ week of gestation, occurring as a result of the failure of development of the cloacal membrane. The cloaca in the embryo is a cavity into which the hindgut (posteriorly) and the allantois (anteriorly) open. The cloacal membrane breaks down creating two openings: the urogenital and the anal ones [5]. The development of each of these parts may be disrupted in the prenatal period, which can have as a consequence the development of various rectal malformations. The classification of Melbourne (1976) of anorectal defects is commonly used. Currently, it has been modified by Pena [6]. It divides the anorectal malformations into low, high and intermediate on the basis of their anatomic position referred to the loop of the puborectalis muscle $[4,6]$. There is a correlation between the localisation of the anorectal abnormalities and the prevalence of concomitant urogenital defects, with the higher malformation that have a greater probability of concomitant urinary tract lesions. In our case the anorectal malformation was localised above the puborectalis muscle. The stool filling the accessory pouch of the rectum, caused recurrent high perineal abscesses (difficult to diagnose and drain) and, finally, led to the formation of an extrasphincteric fistula connecting the cutaneous opening with the rectal duplication. The diagnostic challenge of this condition was due to the multiple treatments of high perineal abscesses in various hospitals, without conducting a diagnostic work-up in order to identify the reason of the recurrent sepsis.

During the ERUS we performed, the diagnosis of rectal malformation (accessory rectum, rectal diverticulum) was suggested. Ultrasonography is highly accurate enabling the visualisation of rectal wall abnormalities and the identification of lesions localised beyond the rectum [7]. ERUS has also an important role in the assessment of anorectal fistulae, determining type, level, branching and internal opening and recurrences of fistulae [8]. MRI confirmed the diagnosis and helped to plan the management. Due to the proximity of the lesion to the rectum and to the urinary tract, the surgery comprised an interdisciplinary team of physicians, including a urologist and colorectal surgeon, experienced in laparoscopic surgery.

In summary, in case of recurrent, atypical anal abscesses or fistulae running beyond the anal sphincters, a comprehensive diagnostic work-up should be performed. ERUS represents the first line of anorectal assessment. MRI can be performed as the second line, in selected cases, to confirm the ultrasound findings. Treating patients with atypical lesions localised in the small pelvis and perianal region is challenging and requires a multidisciplinary team (colorectal surgeon, urologist and radiologist).

\section{References}

1. Tohda A, Hosokawa S, Shimada K. Urinary tract abnormalities associated with anorectal malformations. Nihon Hinyokika Gakkai Zasshi 1995;86:1388-1393.

2. Mittal A, Airon RK, Magu S, Rattan KN, Ratan SK. Associated anomalies with anorectal malformation (ARM). Indian J Pediatr 2004;71:509-514.

3. Santoro GA, Fortling B. The advantages of volume rendering in three-dimensional endosonography of the anorectum. Dis Colon Rectum 2007;50:359-368.

4. Levitt MA, Pena A. Pitfalls in the management of newborn cloacas. Pediatr Surg Int 2005;21:264-269.

5. Gershon M. Endodermal derivatives, formation of the gut and its subsequent rotation. In: Larsen WJ. Human Embriology. 3rd edition. Churchill Livingstone, 2001: 134-149, 235-259. 
6. Pena A, Levitt M.Anorectal malformations. In: Stringer M, Oldham K, Mouriquand PDE. (Eds.). Pediatric Surgery and Urology: Long term outcomes. 2nd edition. Cambridge: Cambridge University Press 2007:401-415.

7. Burdan F, Sudol-Szopinska I, Starosławska E, et al. Magnetic resonance imaging and endorectal ultra- sound for diagnosis of rectal lesions. Eur J Med Res 2015;20:4.

8. Sudoł-Szopińska I, Kucharczyk A, Kołodziejczak M, Warczyńska A, Pracoń G, Wiączek A. Endosonography and magnetic resonance imaging in the diagnosis of high anal fistulae - a comparison. J Ultrason 2014;14:142-151. 